\title{
Le développement collaboratif d'un nouveau concept pour une activité
}

Jaakko Virkkunen

\section{(2) OpenEdition}

1 Journals

Édition électronique

URL : http://journals.openedition.org/activites/1758

DOI : 10.4000/activites. 1758

ISSN : 1765-2723

Éditeur

ARPACT - Association Recherches et Pratiques sur les ACTivités

Référence électronique

Jaakko Virkkunen, «Le développement collaboratif d'un nouveau concept pour une activité », Activités [En ligne], 4-2 | octobre 2007, mis en ligne le 15 octobre 2007, consulté le 20 avril 2019. URL : http:// journals.openedition.org/activites/1758; DOI : 10.4000/activites.1758

\section{(@) $\odot \Theta$}

Activités est mis à disposition selon les termes de la licence Creative Commons Attribution - Pas d'Utilisation Commerciale - Pas de Modification 4.0 International. 


\title{
Le développement collaboratif d'un nouveau concept pour une activité
}

\author{
Jaakko Virkkunen \\ Center for Activity Theory and Developmental Work Research, Departmet of Education, University of Helsinki. \\ P.O. Box 26 FIN-00014 University of Helsinki, Finland
}

\begin{abstract}
In all productive activities in the market economy there is a fundamental inner contradiction between the use value of a produced commodity and its exchange value in the markets. Each technological revolution profoundly changes the conditions of balancing these contradictory demands by enabling the creation of new kinds of use values, the reduction of costs, and a broadened exchange. The culturally available forms of activity can be seen as different concepts of balancing contradictory demands. Currently, the concept of mass production is being replaced by new concepts which utilize the possibilities that the new digital information and communication technologies provide. The creation and implementation of a new concept for an activity is, however, a complex process in which many complementary innovations must be integrated into a functioning whole. In this paper, I will discuss the possibility of applying the principle of collective invention in the creation of a new operating concept.
\end{abstract}

KEYWORDS

Historical types of work, the concept of an activity, activity system, collective invention

\section{1.- Le concept d'une activité}

Le développement des systèmes d'activité de production n'a pas lieu de manière linéaire, mais via des cycles de transformation dans lesquels la logique de l'activité change qualitativement. De tels changements sont invariablement liés à une redéfinition en expansion de l'objet ou du produit de l'activité, autant qu'au développement des nouveaux outils et formes de collaboration associés. Dans l'économie de marché, de telles transformations sont rendues inévitables par l'aggravation du déséquilibre existant entre la valeur d'usage des produits et leur valeur d'échange sur les marchés. Les innovations technologiques radicales, notamment celles qui créent une nouvelle infrastructure de production et d'échanges, changent les conditions de cet équilibre. Ainsi, dans les conditions spécifiques du marché et de la technologie du moment, chaque nouvelle ère engendre un nombre de principes et de logiques spécifiques qui reconfigurent cet équilibre. Ces principes et ces logiques à l'œuvre à un moment donné peuvent être considérés comme des concepts qui fournissent une base garantissant une cohérence mutuelle entre les différents éléments du système de l'activité.

D'après Freeman et Louça (2000), la longue période de croissance économique entre 1945 et 1973 se basait sur l'utilisation d'une énergie peu onéreuse, et sur l'élargissement du panel des moyens de transport engendré par l'invention des moteurs à combustion interne et la technologie de raffinage du pétrole. Cette nouvelle ressource a été utilisée en relation avec l'application du principe de production de masse, qui devint le concept dominant d'organisation des activités de travail à cette époque. Ce concept fut appliqué, mutatis mutandis, dans presque tous les domaines de la production matérielle et des services.

Bart Victor et Andrew Boynton (1998) ont présenté un modèle de la trajectoire de développement des formes de travail - que nous assimilons à des concepts avec notre terminologie (voir Figure 1). D'après eux, une nouvelle étape de développement s'opère toujours sur la base d'une reconfiguration 
des possibilités créées à l'étape précédente, l'enjeu étant d'adapter l'activité aux conditions changeantes du marché.

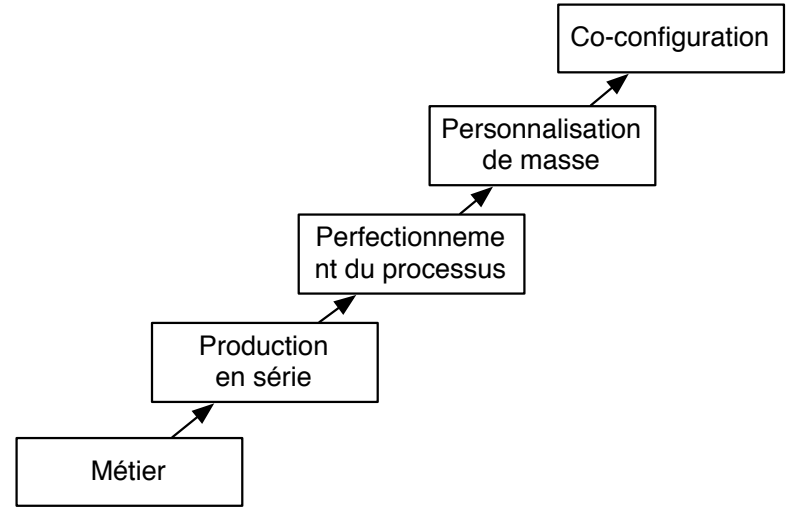

Figure 1: Types historiques d'organisation du travail d'après Victor et Boynton

Victor et Boynton soutiennent que chaque activité locale doit utiliser le « bon chemin » c'est-à-dire celui du développement historique initial des concepts de l'activité. Pour ces auteurs, l'artisanat est à la base de tous les autres, parce qu'il fournit la base du savoir-faire d'un produit spécifique. La transition vers la production de masse devient nécessaire lorsque le volume de l'activité augmente. Il s'agit notamment d'expliquer, de désintégrer, d'optimiser, d'élargir, de standardiser et de réoutiller le procédé de production qui a été créé lors de la période artisanale de l'activité. Les caractéristiques principales du concept de la production de masse sont une organisation séquentielle du travail, une forte spécialisation séquentielle, et une prise de décision hiérarchique et centralisée.

Le concept de démarche qualité, qui permet au fond d'accroître la flexibilité et de dépasser le concept de production de masse, a été développé à l'origine par Toyota 1945-1975 (Ohno, 1978). Il s'est répandu sur le plan international grâce à ses bons résultats dans l'organisation du travail mais aussi parce qu'il correspondait mieux aux conditions des marchés de masse de plus en plus saturés. La démarche qualité ajoute une caractéristique de réciprocité et de retour d'expérience à la logique séquentielle de la production de masse, conduit à regrouper quelques-unes des tâches de production à travers le travail d'équipe, et décentralise la prise de décision.

Les technologies d'information et de communication n'ont pas été centrales dans le développement des concepts comme celui de la production de masse ou celui de la démarche qualité. La segmentation des produits de masse, qui fut développée dans les années 1980, se base cependant déjà dans une grande mesure sur les nouvelles technologies de l'information et de la communication. Elle casse la logique séquentielle linéaire et la spécialisation fonctionnelle encore plus que la démarche qualité, et les remplace par une architecture de la production basée sur une plate-forme de produits et des modules interchangeables. La production est réalisée par divers procédés dans un réseau dynamique de coopération.

Comme décrit par Victor et Boynton (1998), le concept le plus récent de l'activité, la co-configuration, est encore plus basé sur l'utilisation des nouvelles technologies de l'information et de la communication. Il est caractérisé par: 1) un produit tourné vers le client qui peut être adapté de manière continuelle, pour une part par le producteur et partiellement par l'utilisateur, aux conditions changeantes et aux besoins des clients, 2) un système collaboratif de création de valeur, dans lequel la valeur n'est pas produite dans l'activité du producteur ni dans l'activité de l'utilisateur, mais dans l'interaction et la collaboration existant entre eux, 3) l'adaptation continuelle: le producteur n'adapte pas le produit ou le service ponctuellement mais de manière continuelle, et le met à jour par exemple par le biais des mises à jour logicielles. Ainsi, le concept de co-configuration entraîne un dialogue continu et orienté vers le développement entre le producteur et l'utilisateur. Au sein de l'organisation de production, il casse la logique séquentielle traditionnelle de recherche, puis de développement du produit, puis de production et de mise sur marché. Ces phases deviennent plutôt des aspects concomitants au sein de la collaboration continue 
existant entre le producteur et l'utilisateur. Dans la coopération «B to B », une part du développement de produit est réalisée avec le client au sein d'une collaboration étroite dans laquelle les deux partenaires apprennent l'un de l'autre et de la collaboration elle-même.

Le type de collaboration continuellement reconfigurée et orientée vers le développement entre les organisations spécialisées que Victor et Boyton décrivent peut être vu comme un nouveau type de concept d'activité de l'ère de l'information. Plus généralement, il semble que, au sein de nouveaux concepts évolutifs, la logique séquentielle et la division fonctionnelle rigide du travail typique de la production de masse sont progressivement remplacées ou complétées par des formes de dialogue, par la réalisation concomitante de différents aspects de la même activité, et par de nouvelles manières d'intégrer les connaissances et les fonctions spécialisées afin de maîtriser des objets de plus en plus complexes.

\section{2.- Le développement d'un nouveau concept de l'activité}

D'après le modèle de Victor et Boynton, une activité peut être développée au sein du concept dominant: artisanat comme artisanat, production de masse comme production de masse, etc., ou le concept de l'activité peut être transformé: de l'artisanat à la production de masse, de la production de masse à la démarche qualité, etc. Ces derniers peuvent être vus comme des apprentissages collectifs lors un processus d'expansion (Engeström, 1987), dans lequel un nouveau concept est créé et mis en œuvre pour surmonter une impasse de développement et une contradiction intrinsèque s'aggravant au sein du concept prédominant. Ces processus sont typiquement des processus complexes et construits sur le long terme, dans lesquels les acteurs rencontrent continuellement des nouvelles contradictions à surmonter au sein de l'activité. Par exemple, l'évolution du système de production Toyota (et le concept de démarche qualité) a duré trente ans d'après un décompte réalisé par l'ingénieur en chef Taiichi Ohno (1978). Pendant ces années, plusieurs innovations majeures et d'innombrables petites innovations ont été faites sur différents aspects de l'activité de production, telle que l'installation, la maniabilité de la machine, la coordination entre différentes phases de la production, le développement de la communication entre les acteurs, et les logistiques internes.

La durée et la complexité du développement d'un nouveau concept induisent un problème particulier: maintenir la continuité du processus de développement d'un concept. Comme le note Vygotsky (1986), un problème ou un objectif ne suffit pas à initier un processus d'élaboration conceptuelle, même s'il constitue nécessairement un prérequis. Il est également nécessaire qu'il existe une sorte de symbole permettant d'aider les acteurs à focaliser leur attention et leur fournissant une direction pour le développement du nouveau concept. «Le développement et l'utilisation de stimuli artificiels jouent un rôle d'auxiliaire, de point d'appui, qui permet aux êtres humains de maitriser leur propre comportement, d'abord par des moyens externes et ensuite par des opérations internes plus complexes » (Vygotsky, 1978). Cette idée ne s'applique pas seulement aux individus mais aussi aux groupes et aux collectifs.

Quand il s'agit de mettre en œuvre un concept connu, plusieurs représentations du concept peuvent être utilisées comme autant d'outils intellectuels afin de guider le processus de transformation. Lorsqu'un nouveau concept est développé, la cohérence du processus de développement ne peut pas être atteinte de cette manière, puisque le contenu de ce nouveau concept n'est pas encore connu. Un symbole ou une représentation sont nécessaires, qui incarneront de manière paradoxale ce qu'on ne connaît pas encore et ce qui devra (qui pourra probablement) être appris ou inventé (Rheinberger, 1997). Une manière de faire est d'identifier une contradiction qui devra être surmontée au sein du système de l'activité. Dans le développement du système de production Toyota, par exemple, il s'agissait d'abord de la contradiction croissante entre le concept de la production de masse et les petits marchés au Japon. Ensuite, comme Fujimoto (1999) le décrit, il existait aussi une contradiction croissante entre, d'une part, la recherche d'une bonne qualité, de coûts moindres et d'une « production juste à temps », et d'autre part la fai- 
ble motivation des travailleurs et leur bien-être insuffisant visible dans un fort niveau turnover.

\section{3.- Collaboration inter-entreprises lors du développement d'un nouveau concept d'activité}

Une façon d'accélérer le développement de nouveaux concepts pour les activités est de favoriser la collaboration horizontale pour le développement entre des entreprises ou des unités qui réalisent le même type d'activité. Nuovolari et Mayer (Nuovolari, 2001; Mayer, 2003) ont décrit plusieurs processus d'invention collective dans l'histoire de l'industrie - le dernier étant le développement du logiciel libre: les utilisateurs compétents, qui utilisent le même système technique dans différents contextes, échangent les améliorations qu'ils réalisent lors de l'usage du logiciel. En conséquence, la technologie devient plus robuste et une plus grande variété d'applications est créée pour différents usages. La possibilité de réalisation de l'invention collective est basée sur une combinaison de similitudes et de variations qui rendent l'échange possible: la technologie est la même, mais les utilisateurs trouvent des problèmes et des possibilités de développement différents parce qu'ils l'appliquent dans des contextes ou pour des objectifs différents.

Lorsque le même concept est appliqué au sein de différentes activités locales, les problèmes relatifs à son utilisation ainsi que les solutions potentielles sont également les mêmes. Les concepts dominants d'activité sont dans la plupart des cas basés sur les concepts qui ont évolué pendant la longue période de production de masse d'après-guerre. Le changement dans les dynamiques de développement industriel conduit désormais beaucoup de ces concepts à un point critique. Pour diverses occurrences locales d'une même activité, il existe des points communs dans les contradictions apparues historiquement. Cela fournit une base objective pour l'invention collective lors du développement d'un nouveau concept d'activité.

Les activités locales rencontrent néanmoins de nouveaux enjeux à différents moments, les acteurs impliqués formulent diverses hypothèses concernant le moyen de surmonter les contradictions et ils suivent différentes voies pour se faire. C'est pourquoi le point de convergence des contradictions basiques existant au sein de systèmes parallèles d'activités est masqué par différents problèmes aigus et différentes stratégies de développement des activités locales. La reconnaissance du point de convergence entre les contradictions de développement sous-jacentes aux problèmes aigus doit être investiguée par le biais d'un processus spécial d'analyse et de modélisation, afin de rendre possibles les échanges et l'invention collective pour le développement d'un nouveau concept. Basée sur la théorie de l'activité, la méthodologie de recherche sur le travail de développement (Developmental Work Research) (Engeström, 1987) peut être utile dans la réalisation d'un tel processus. Associé à l'analyse historique et empirique de l'activité locale, le modèle général du système d'activité d'Engeström (1987) peut être utilisé pour modéliser le concept d'activité antérieur, et les contradictions internes actuelles résultant des évolutions récentes de ses éléments constitutifs (voir Figure 2).

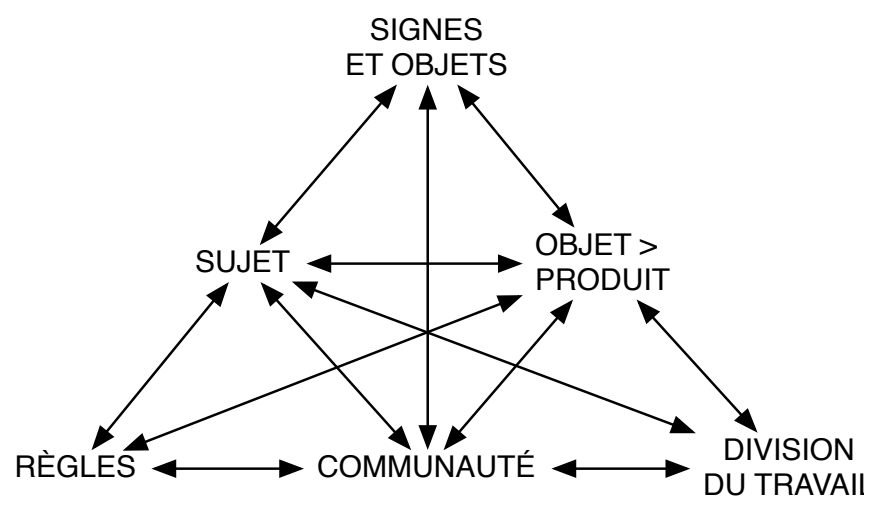

Figure 2 : Le modèle général du système d'activité 
Dans ce modèle, un acteur de l'activité est un membre d'une communauté d'acteurs travaillant sur le même objet pour créer un produit. Les actions individuelles, de même que les nécessaires échanges et distributions de produits intermédiaires et de ressources, sont réalisés par le biais d'outils, de règles, et de la division du travail inhérents au système d'activité.

La transformation du concept d'une activité de travail collaboratif est un processus progressif et compliqué de remédiation, dans lequel le changement d'un des médiateurs de l'activité crée de nouvelles contradictions internes au sein de chaque élément et entre les éléments du système de l'activité. Ces premières remédiations vont à leur tour rendre nécessaires de nouvelles remédiations jusqu'à transformation de l'ensemble du système d'activité. Le processus cyclique d'expansion et de transformation globale du concept d'une activité tire donc sa force motrice d'une succession d'émergences et de résolutions de nouveaux types de contradictions internes au sein du système de l'activité (voir Figure 3).

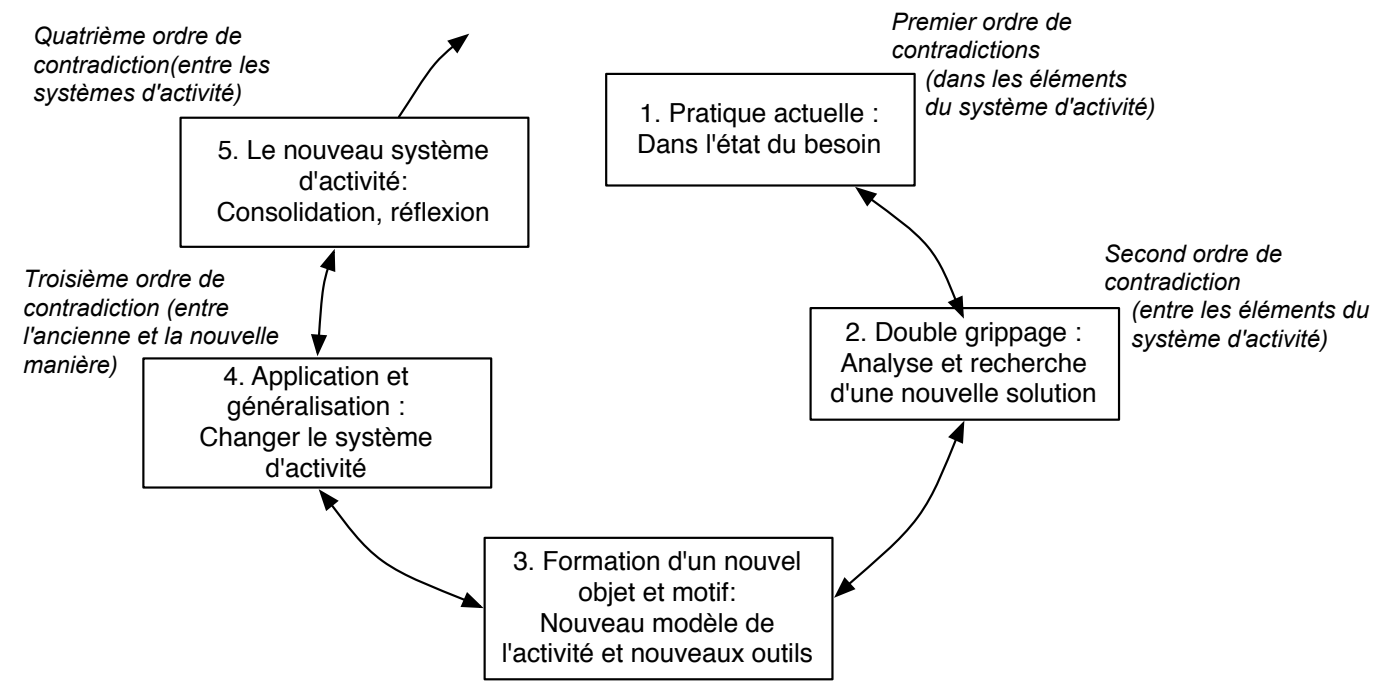

Figure 3: Le cycle d'expansion du concept d'une activité

La première phase du cycle est caractérisée par une aggravation graduelle de la contradiction de premier ordre existant entre la valeur d'usage et la valeur d'échange. Lorsque d'autres éléments changent au sein de l'activité, l'état provisoirement atteint se développe par l'apparition de contradictions secondaires entre les éléments du système. Ces contradictions se manifestent dans des situations inextricables pour les acteurs individuels. L'adoption d'un nouveau médiateur, qui redéfinit l'objet et le produit de l'activité, initie le processus de transformation du système d'activité. Provoquées par les collisions entre les nouveaux et les anciens éléments de l'activité, de nouvelles innovations apparaissent. Dans la dernière phase du cycle d'expansion de l'activité via des remédiations, de nouveaux médiateurs doivent aussi être créés pour assurer l'interaction entre l'activité centrale et les activités périphériques dans le réseau qui existe entre différents systèmes d'activité.

Bien que plusieurs occurrences locales de la même activité puissent partager la contradiction interne basique, elles peuvent cependant se trouver dans une phase différente du processus de transformation de l'activité et de création d'un nouveau concept. Le modèle idéal-typique d'Engeström (1987) concernant le processus cyclique d'expansion et de transformation globale du concept d'une activité, détaillé dans la figure 3, fournit un moyen de modéliser les différentes phases de transformation d'activités parallèles, et, se faisant, aide les acteurs à voir qu'ils accomplissent une transformation similaire, même s'ils ne se trouvent pas dans la même phase.

Au sein d'un réseau expérimental formé de personnes engagées dans le développement du travail, nous testons actuellement l'hypothèse suivante: l'invention collective pendant le développement d'un nouveau concept d'activité peut être favorisée en fournissant aux acteurs 
des exemples d'occurrences localement situés de l'activité en vue de les aider à modéliser les contradictions centrales de leur activité et à identifier la phase actuelle de développement de l'activité au sein du cycle global d'expansion. À travers ce type de modélisation, nous souhaitons que les acteurs identifient les points de convergence derrière les divers problèmes apparents, et ainsi trouvent une base d'échange des points de vue et des innovations partielles pour développer le nouveau concept.

\section{RÉFÉRENCEMENT}

Virkkunen, J. (2007). Le dévelopement collaboratif d'un nouveau concept pour une activité. @ctivités, 4 (2), pp. 151-157, http://www.activites.org/v4n2/v4n2.pdf

\section{BIBLIOGRAPHIE}

Engeström, Y. Learning by expanding: An activity-theoretical approach to developmental research, 1987. Availble at: http://lchc.ucsd.edu/MCA/Paper/Engetrom/expanding /toc.

Freeman, C., \& Louça, F. (2000). As Time Goes By. Oxford: Oxford University Press.

Fujimoto, T. (1999). The evolution of a manufacturing system at Toyota. New York: Oxford University Press.

Mayer, P. M. (2003). Episodes of collective invention. U.S Department of Labor. Bureau of Labor Statistics. Working paper 368, 2003.

Nuovolari, A. (2001). Collective invention during the British industrial revolution. The case of the Cornish pumping engine. Danish Research Unit for Industrial Dynamics. Working Paper 01-05, 2001.

Ohno,T. (1978). The Toyota Production System. Beyond large scale production. Portland Oregon: The Productivity Press.

Rheinberger, H.J. (1997). Toward a history of epistemic things. Synthesizing proteins in the test tube. Stanford California: Stanford University Press.

Victor, B., \& Boynton. A. (1998). Invented Here: Maximizing Your Organization's Internal Growth and Profitability. A Practical Quide to Transforming Work. Boston, Mass.: Harvard Business School Press.

Vygotsky, L. S. (1978). Mind in society. Cambridge, Mass.: Harvard University Press.

Vygotsky, L. S. (1986). Thought and Language. Cambridge Mass.: The MIT Press.

\section{RÉSUMÉ}

Au sein de l'économie de marché, pour toute activité de production, il existe une contradiction intrinsèque entre la valeur d'usage du produit et sa valeur d'échange sur les marchés. Chaque révolution technologique change profondément les conditions d'équilibre de cette contradiction, en permettant la création de nouvelles valeurs d'usage, en offrant des possibilités de réduction des coûts, et en favorisant un élargissement des échanges. Les formes d'activité culturellement disponibles peuvent être considérées comme différents concepts qui correspondent à des équilibres différents de cette contradiction. Actuellement, le concept de production de masse est progressivement remplacé par de nouveaux concepts qui se développent aussi à la faveur de l'exploitation des possibilités fournies par les nouvelles technologies de l'information et de la communication. La création et la mise en œuvre d'un nouveau concept pour une activité est malgré tout un processus complexe, dans lequel beaucoup d'innovations complémentaires doivent être intégrées en un tout fonctionnel. Dans cet article, nous nous proposons de discuter de la possibilité d'appliquer le principe de l'invention collective à la création d'un nouveau concept pour la production. 
MotS ClÉS

Types d'organisation du travail, concept d'activité, système d'activité, invention collective

\section{RESUMEN}

En toda actividad de producción de la economía de mercado, existe una contradicción intrínseca fundamental entre el valor de uso de un comodity producido y su valor de intercambio en el mercado. Cada revolución tecnológica cambia profundamente las condiciones de equilibrio de esta contradicción, permitiendo la creación de nuevos valores de uso, ofreciendo posibilidades de reducción de costos, y favoreciendo la ampliación de los intercambios. Las formas de actividad culturalmente disponibles pueden ser consideradas como diferentes conceptos acerca de cómo se logran equilibrar diferentes exigencias. Actualmente, el concepto de producción en masa está siendo reemplazado por nuevos conceptos que utilizan las posibilidades ofrecidas por las nuevas tecnologías de la información y de la comunicación. La creación e implementación de un nuevo concepto para una actividad es, sin embargo, un proceso complejo en el cual múltiples innovaciones complementarias deben integrarse en un todo funcional. En este artículo, discutiré la posibilidad de aplicar el principio de invención colectiva a la creación de un nuevo concepto operativo/operacional.

Palabras clave

Tipos de trabajo históricos, el concepto de una actividad, sistema de actividad, invención colectiva 